POS PROCEEDINGS

\title{
Landau gauge gluon and ghost propagators from two-flavor lattice QCD at $T>0$
}

\section{R. Aouane, F. Burger, M. Müller-Preussker*,}

Humboldt-Universität zu Berlin, Institut für Physik, 12489 Berlin, Germany

\section{E.-M. Ilgenfritz}

Joint Institute for Nuclear Research, VBLHEP, 141980 Dubna, Russia

\section{A. Sternbeck}

Universität Regensburg, Institut für Theoretische Physik, 93040 Regensburg, Germany

In this contribution we extend our unquenched computation of the Landau gauge gluon and ghost propagators in lattice QCD at non-zero temperature. The study was aimed at providing input for investigations employing continuum functional methods. We show data which correspond to pion mass values between 300 and $500 \mathrm{MeV}$ and are obtained for a lattice size $32^{3} \times 12$. The longitudinal and transversal components of the gluon propagator turn out to change smoothly through the crossover region, while the ghost propagator exhibits only a very weak temperature dependence. For a pion mass of around $400 \mathrm{MeV}$ and the intermediate temperature value $T \simeq 240 \mathrm{MeV}$ we compare our results with additional data obtained on a lattice with smaller Euclidean time extent $N_{\tau}=$ 8,10 and find a reasonable scaling behavior.

The 31 International Symposium on Lattice Field Theory - Lattice 2013,

July 29 - August 3, 2013

Mainz, Germany

\footnotetext{
*Speaker.
} 


\section{Introduction}

Over the last 15 years Dyson-Schwinger (DS) equations and functional renormalization group (FRG) equations turned into powerful schemes to address various nonperturbative problems in QCD. Within this framework (for reviews see e.g. [1, 2]) the Landau gauge gluon and ghost propagators appear - together with the corresponding vertices - as the main building blocks. In order to solve the (infinite) tower of corresponding equations an appropriate truncation has to be applied. Then independent information, preferably from first principles, is welcome to improve the resulting approximations or to be used as an input to the system of equations.

Lattice QCD calculations allow to provide e.g. the Landau gauge gluon and ghost propagators in an ab-initio way. The available momentum range, however, is restricted from above by the lattice spacing and from below by the available lattice volume up to a further uncertainty related to so-called Gribov copies (see e.g. [3] and references therein).

In recent years the DS / FRG framework has been applied also to explore the nonzero temperature regime of QCD and even the non-zero chemical potential case (see [4] and references therein).

Correspondingly we have started a lattice computation of gluon and ghost propagators at non-zero temperature, first in $S U$ (3) pure gauge theory [5] and more recently for the full QCD case with two light flavor degrees of freedom [6]. Here we report on the latter case. For that we could rely on investigations of the QCD thermodynamics employing the Wilson-twisted mass discretization at maximal twist [7, 8]. For three pion mass values smooth crossover regimes were found, where the signals for the transition taken from the (renormalized) Polyakov loop and from the chiral condensate (susceptibility) occured at different temperature values $T_{\text {deconf }}$ and $T_{\chi}$, respectively.

Throughout those crossover regions we have obtained the momentum dependence of the transverse und longitudinal components of the Landau gauge gluon propagator as well as of the corresponding ghost propagator. Additionally to the results published in [6] we add a first scaling test in order to check, in as far we are close to the continuum limit in the momentum range considered.

\section{Observables}

Our first quantity of interest is the gluon propagator in momentum space

$$
D_{\mu v}^{a b}(q)=\left\langle\widetilde{A}_{\mu}^{a}(k) \widetilde{A}_{v}^{b}(-k)\right\rangle
$$

where $\langle\cdots\rangle$ represents the path integral average estimated by averaging over gauge-fixed (Hybrid) Monte Carlo generated gauge field configurations. $\widetilde{A}_{\mu}^{a}(k)$ denotes the Fourier transform of the gauge potential. $k_{\mu} \in\left(-N_{\mu} / 2, N_{\mu} / 2\right]$ is the lattice momentum $(\mu=1, \ldots, 4)$ related to the physical momentum via $q_{\mu}\left(k_{\mu}\right)=\frac{2}{a} \sin \left(\frac{\pi k_{\mu}}{N_{\mu}}\right)$. We use the notation $\left(N_{\sigma} ; N_{\tau}\right) \equiv$ $\left(N_{i} ; N_{4}\right)$ where $i=1,2,3$. 
The gauge is fixed with the help of a simulated annealing prescription to extremize the Landau gauge functional always followed by overrelaxation steps until a prescribed numerical precision for the local gauge condition is reached. For a discussion about the efficiency of the simulated annealing procedure in Landau gauge fixing see [9].

For non-zero temperature the Euclidean invariance is broken. Therefore, it is useful to split $D_{\mu v}^{a b}(q)$ into two components, the transversal $D_{T}$ ("chromomagnetic") and the longitudinal $D_{L}$ ("chromoelectric") propagator, respectively. For $D_{T, L}$ [or their respective dimensionless dressing functions $Z_{T, L}(q)=q^{2} D_{T, L}(q)$ ] one finds (see also [5])

$$
D_{T}(q)=\frac{1}{2 N_{g}}\left\langle\sum_{i=1}^{3} \widetilde{A}_{i}^{a}(k) \widetilde{A}_{i}^{a}(-k)-\frac{q_{4}^{2}}{\vec{q}^{2}} \widetilde{A}_{4}^{a}(k) \widetilde{A}_{4}^{a}(-k)\right\rangle
$$

and

$$
D_{L}(q)=\frac{1}{N_{g}}\left(1+\frac{q_{4}^{2}}{\vec{q}^{2}}\right)\left\langle\widetilde{A}_{4}^{a}(k) \widetilde{A}_{4}^{a}(-k)\right\rangle,
$$

where $N_{g}=N_{c}^{2}-1$ and $N_{c}=3$.

The zero-momentum gluon propagator values can be defined as

$$
\begin{aligned}
& D_{T}(0)=\frac{1}{3 N_{g}} \sum_{i=1}^{3}\left\langle\widetilde{A}_{i}^{a}(0) \widetilde{A}_{i}^{a}(0)\right\rangle, \\
& D_{L}(0)=\frac{1}{N_{g}}\left\langle\widetilde{A}_{4}^{a}(0) \widetilde{A}_{4}^{a}(0)\right\rangle .
\end{aligned}
$$

The Landau gauge ghost propagator is given by

$$
\begin{aligned}
G^{a b}(q) & =a^{2} \sum_{x, y}\left\langle e^{-2 \pi i(k / N) \cdot(x-y)}\left[M^{-1}\right]_{x y}^{a b}\right\rangle \\
& =\delta^{a b} G(q)=\delta^{a b} J(q) / q^{2}
\end{aligned}
$$

where $(k / N) \equiv\left(k_{\mu} / N_{\mu}\right) . J(q)$ denotes the ghost dressing function. The matrix $M$ is the lattice Faddeev-Popov operator, for more details see [5]. For the inversion of $M$ we use the pre-conditioned conjugate gradient algorithm of [10] with plane-wave sources $\vec{\psi}_{c}$ with color and position components $\psi_{c}^{a}(x)=\delta_{c}^{a} \exp (2 \pi i k \cdot(x / N))$.

In order to reduce lattice artifacts we restrict ourselves to diagonal and slightly offdiagonal momenta for the gluon propagator and diagonal momenta for the ghost propagator. Moreover, only modes with zero Matsubara frequency $\left(k_{4}=0\right)$ are considered.

Assuming that the influence of lattice artifacts can be neglected the renormalized dressing functions, defined in momentum subtraction (MOM) schemes, can be obtained via multiplicative renormalization

$$
\begin{aligned}
Z_{T, L}^{r e n}(q, \mu) & \equiv \tilde{Z}_{T, L}(\mu) Z_{T, L}(q), \\
J^{r e n}(q, \mu) & \equiv \tilde{Z}_{J}(\mu) J(q)
\end{aligned}
$$

with the $\tilde{Z}$-factors being defined such that $Z_{T, L}^{r e n}(\mu, \mu)=J^{r e n}(\mu, \mu)=1$. Our renormalization scale throughout this paper is $\mu=2.5 \mathrm{GeV}$ (see also Ref. [6]). 

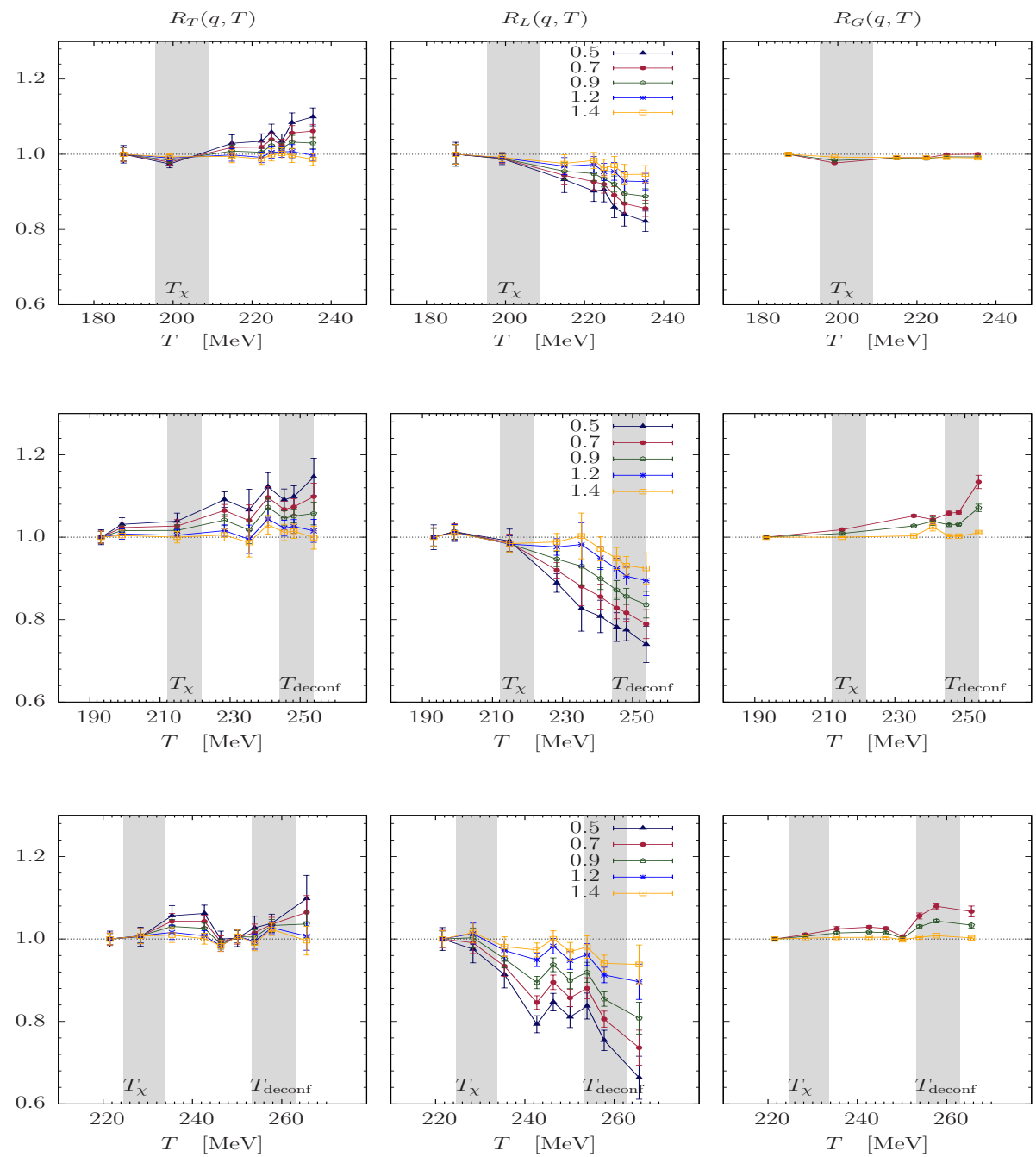

Figure 1: Ratios $R_{T}, R_{L}$ and $R_{G}$ for the renormalized transverse $D_{T}^{r e n}$ (left panel), longitudinal $D_{L}^{r e n}$ (middle panel) and ghost $G^{r e n}$ (right panel) propagators, respectively, as functions of the temperature $T$ at a few non-zero momentum values $q$ (indicated in units of $[\mathrm{GeV}]$. The corresponding pion masses (from top to bottom) are $m_{\pi} \simeq 316,398$ and $469 \mathrm{MeV}$. The vertical bands indicate the chiral $\left(T_{\chi}\right)$ and 'deconfinement' $\left(T_{\text {deconf }}\right)$ pseudo-critical temperatures with their uncertainties.

\section{Results}

Our estimates for $D_{T, L}$ and $G(q, T)$ rely on gauge field ensembles generated on a lattice of size $N_{\sigma}=32$ and $N_{\tau}=12$ and for parameters which are collected in detail in [6].

In Fig. 1 we show ratios of the renormalized dressing functions or propagators

$$
\begin{aligned}
R_{T, L}(q, T) & =D_{T, L}^{r e n}(q, T) / D_{T, L}^{r e n}\left(q, T_{\min }\right), \\
R_{G}(q, T) & =G^{r e n}(q, T) / G^{r e n}\left(q, T_{\min }\right)
\end{aligned}
$$

as functions of the temperature $T$ for 6 fixed (interpolated) momentum values $q \neq 0$, and for the three different pion masses (panels from top to bottom). For better visibility, ratios are normalized with respect to the respective left-most shown temperature $T_{\min }$ in Fig. 1. 

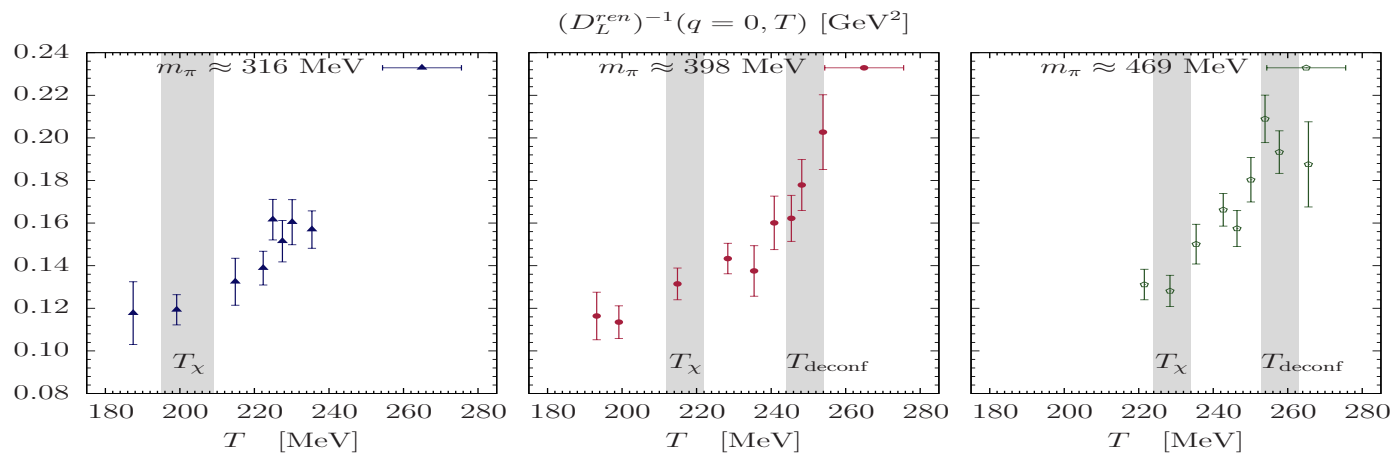

Figure 2: The panels show the inverse renormalized longitudinal gluon propagator $\left(D_{L}^{r e n}\right)^{-1}$ at zero momentum for the three pion mass values indicated.
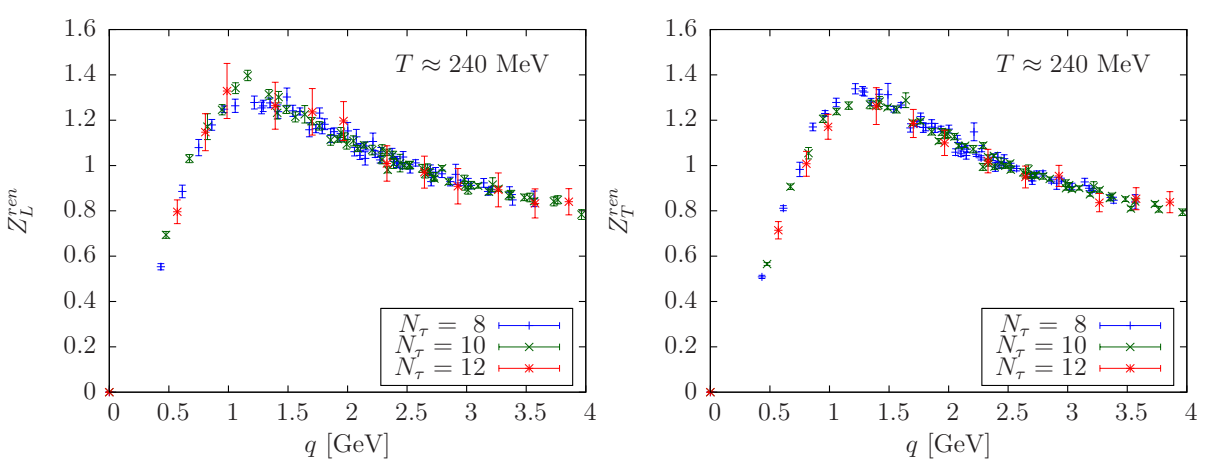

Figure 3: The left (right) panel shows the renormalized longitudinal (transversal) gluon dressing function for a scaling check by varying $N_{\tau}=8,10,12$ at $m_{\pi} \simeq 398 \mathrm{MeV}$ and $T \simeq 240 \mathrm{MeV}$.

We see $R_{L}(q, T)$ to decrease more or less monotonously with the temperature through the crossover region, and this decrease is stronger the smaller the momentum is. This behavior is similar but much less pronounced than what we saw in the close neighborhood of the phase transition for the quenched case [5], where a first-order phase transition takes place.

$R_{T}(q, T)$ instead signals a slight increase within the same range, and the ghost propagator (at fixed low momenta) seems to rise near $T \simeq T_{\text {deconf. }}$.

The panels of Fig. 2, show data for the inverse renormalized longitudinal propagator $1 / D_{L}^{r e n}$ at zero momentum, again versus temperature and separately for different pion masses. Assuming a linear $q^{2}$-behavior as $D_{L}^{r e n}(q, T)^{-1} \sim q^{2}+m_{s c}(T)^{2}$ at low $q^{2}$, this quantity can be related to a gluon screening mass $m_{s c}(T)$. Since $D_{L}^{r e n}(0)^{-1}$ rises with temperature in the crossover region it may also serve as a useful indicator for the finite-temperature crossover of the quark-gluon system. However, such zero-momentum results are always influenced by strong finite-size and Gribov copy effects, which we have not analyzed here.

Finally in Fig. 3 we show a scaling test achieved with gauge field ensembles produced additionally at smaller time-like lattice extent $N_{\tau}$ but at approximately the same values of the charged pion mass $\left(m_{\pi} \simeq 398 \mathrm{MeV}\right)$ and temperature $T \simeq 240 \mathrm{MeV}$ inside the crossover region. We see that the results for the two largest values $N_{\tau}$ nicely agree 
within the achieved statistical errors telling us that the $N_{\tau}=12$ results seem already to be close to the continuum limit. For the latter results, in Ref. [6] we have provided fit formulae working reasonably well in the momentum range $0.4 \mathrm{GeV} \leq q \leq 3.0 \mathrm{GeV}$ and hopefully being useful as input for the continuum DS or FRG framework.

We thank the HLRN supercomputing centers Berlin/Hannover for providing us with the necessary computing resources. F.B. and M.M.P. acknowledge support from DFG with an SFB/TR9 grant and R.A. from the Yousef Jameel Foundation at Humboldt-University Berlin. A.S. acknowledges support by the European Reintegration Grant (FP7-PEOPLE2009-RG, No.256594).

\section{References}

[1] C. S. Fischer, A. Maas, and J. M. Pawlowski, "On the infrared behavior of Landau gauge Yang-Mills theory," Annals Phys. 324 (2009) 2408, arXiv: 0810.1987 [hep-ph] .

[2] P. Boucaud, J. Leroy, A. L. Yaouanc, J. Micheli, O. Pene, et al., "The infrared behaviour of the pure Yang-Mills Green functions," Few Body Syst. 53 (2012) 387, arXiv: 1109.1936 [hep-ph] .

[3] A. Sternbeck and M. Müller-Preussker, "Lattice evidence for the family of decoupling solutions of Landau gauge Yang-Mills theory," arXiv:1211.3057 [hep-lat] .

[4] C. S. Fischer and J. Lücker, "Propagators and phase structure of $\mathrm{Nf}=2$ and $\mathrm{Nf}=2+1 \mathrm{QCD}$," arXiv:1206.5191 [hep-ph].

[5] R. Aouane, V. Bornyakov, E. Ilgenfritz, V. Mitrjushkin, M. Müller-Preussker, and A. Sternbeck, "Landau gauge gluon and ghost propagators at finite temperature from quenched lattice QCD," Phys.Rev. D85 (2012) 034501, arXiv:1108.1735 [hep-lat] .

[6] R. Aouane, F. Burger, E.-M. Ilgenfritz, M. Müller-Preussker, and A. Sternbeck, "Landau gauge gluon and ghost propagators from lattice $\mathrm{QCD}$ with $\mathrm{Nf}=2$ twisted mass fermions at finite temperature," Phys.Rev. D87 (2013) 114502, arXiv:1212.1102 [hep-lat] .

[7] tmfT Collaboration, F. Burger, E.-M. Ilgenfritz, M. Kirchner, M. Lombardo, M. Müller-Preussker, O. Philipsen, C. Urbach, and L. Zeidlewicz, "The thermal QCD transition with two flavours of twisted mass fermions," Phys.Rev. D87 (2013) 074508 , arXiv:1102.4530 [hep-lat].

[8] tmfT Collaboration, F. Burger, M. Kirchner, M. Müller-Preussker, E.-M. Ilgenfritz, M. P. Lombardo, et al., "Pseudo-Critical Temperature and Thermal Equation of State from $N_{f}=2$ Twisted Mass Lattice QCD," PoS LATTICE2012 (2012) 068, arXiv: 1212.0982 [hep-lat] .

[9] I. L. Bogolubsky, V. G. Bornyakov, G. Burgio, E.-M. Ilgenfritz, V. K. Mitrjushkin, M. Müller-Preussker, and P. Schemel, "The Landau gauge gluon propagator: Gribov problem and finite-size effects," PoS LAT2007 (2007) 318, arXiv:0710.3234 [hep-lat ] .

[10] A. Sternbeck, E.-M. Ilgenfritz, M. Müller-Preussker, and A. Schiller, "Towards the infrared limit in SU(3) Landau gauge lattice gluodynamics," Phys. Rev. D72 (2005) 014507, hep-lat/0506007. 